\title{
On the Homogeneity of Global Minimizers for the Mumford-Shah Functional when $K$ is a Smooth Cone
}

\author{
Antoine Lemenant (*)
}

Abstract - We show that if $(u, K)$ is a global minimizer for the Mumford-Shah functional in $\mathbb{R}^{N}$, and if $K$ is a smooth enough cone, then (modulo constants) $u$ is a homogenous function of degree $\frac{1}{2}$. We deduce some applications in $\mathbb{R}^{3}$ as for instance that an angular sector cannot be the singular set of a global minimizer, that if $K$ is a half-plane then $u$ is the corresponding cracktip function of two variables, or that if $K$ is a cone that meets $S^{2}$ with an union of $C^{\infty}$ curvilinear convex polygones, then it is a $\mathrm{P}, \mathrm{Y}$ or $\mathrm{T}$.

\section{Introduction.}

The functional of D. Mumford and J. Shah [18] was introduced to solve an image segmentation problem. If $\Omega$ is an open subset of $\mathbb{R}^{2}$, for example a rectangle, and $g \in L^{\infty}(\Omega)$ is an image, one can get a segmentation by minimizing

$$
J(K, u):=\int_{\Omega \backslash K}|\nabla u|^{2} d x+\int_{\Omega \backslash K}(u-g)^{2} d x+H^{1}(K)
$$

over all the admissible pairs $(u, K) \in \mathcal{A}$ defined by

$$
\mathcal{A}:=\left\{(u, K) ; K \subset \Omega \text { is closed }, u \in W_{l o c}^{1,2}(\Omega \backslash K)\right\} .
$$

Any solution $(u, K)$ that minimizes $J$ represents a "smoother" version of the image and the set $K$ represents the edges of the image.

Existence of minimizers is a well known result (see for instance [11]) using $S B V$ theory.

(*) Indirizzo dell'A.: Université Paris XI, Bureau 15 Bâtiment 430, Orsay, 91400, France.

E-mail: antoine.lemenant@math.u-psud.fr 
The question of regularity for the singular set $K$ of a minimizer is more difficult. The following conjecture is currently still open.

CONJECTURE 1 (Mumford-Shah). [18] Let $(u, K)$ be a reduced minimizer for the functional $J$. Then $K$ is the finite union of $C^{1}$ arcs.

The term "reduced" just means that we cannot find another pair $(\tilde{u}, \tilde{K})$ such that $K \subset \tilde{K}$ and $\tilde{u}$ is an extension of $u$ in $\Omega \backslash \tilde{K}$.

Some partial results are true for the conjecture. For instance it is known that $K$ is $C^{1}$ almost everywhere (see [7], [4] and [2]). The closest to the conjecture is probably the result of A. Bonnet [4]. He proves that if $(u, K)$ is a minimizer, then every isolated connected component of $K$ is a finite union of $C^{1}$-arcs. The approach of A. Bonnet is to use blow up limits. If $(u, K)$ is a minimizer in $\Omega$ and $y$ is a fixed point, consider the sequences $\left(u_{k}, K_{k}\right)$ defined by

$$
u_{k}(x)=\frac{1}{\sqrt{t_{k}}} u\left(y+t_{k} x\right), \quad K_{k}=\frac{1}{t_{k}}(K-y), \quad \Omega_{k}=\frac{1}{t_{k}}(\Omega-y) .
$$

When $\left\{t_{k}\right\}$ tends to infinity, the sequence $\left(u_{k}, K_{k}\right)$ may tend to a pair $\left(u_{\infty}, K_{\infty}\right)$, and then $\left(u_{\infty}, K_{\infty}\right)$ is called a Global Minimizer. Moreover, A. Bonnet proves that if $K_{\infty}$ is connected, then $\left(u_{\infty}, K_{\infty}\right)$ is one of the list below:

- 1ST CASE: $K_{\infty}=\varnothing$ and $u_{\infty}$ is a constant.

- 2ND CASE: $K_{\infty}$ is a line and $u_{\infty}$ is locally constant.

- 3RD CASE: "Propeller": $K_{\infty}$ is the union of 3 half-lines meeting with 120 degrees and $u_{\infty}$ is locally constant.

- 4TH CASE: "Cracktip": $K_{\infty}=\{(x, 0) ; x \leq 0\}$ and $u_{\infty}(r \cos (\theta), r \sin (\theta))=$ $= \pm \sqrt{\frac{2}{\pi}} r^{1 / 2} \sin \frac{\theta}{2}+C$, for $r>0$ and $|\theta|<\pi(C$ is a constant), or a similar pair obtained by translation and rotation.

We don't know whether the list is complete without the hypothesis that $K_{\infty}$ is connected. This would give a positive answer to the Mumford-Shah conjecture.

The Mumford-Shah functional was initially given in dimension 2 but there is no restriction to define Minimizers for the analogous functional in $\mathbb{R}^{N}$. Then we can also do some blow-up limits and try to think about what should be a global minimizer in $\mathbb{R}^{N}$. Almost nothing is known in this direction and this paper can be seen as a very preliminary step. Let state some definitions. 
Definition 2. Let $\Omega \subset \mathbb{R}^{N},(u, K) \in \mathcal{A}$ and $B$ be a ball such that $\bar{B} \subset \Omega$. $A$ competitor for the pair $(u, K)$ in the ball $B$ is a pair $(v, L) \in \mathcal{A}$ such that

$$
\left.\begin{array}{rl}
u & =v \\
K & =L
\end{array}\right\} \text { in } \Omega \backslash B
$$

and in addition if $x$ and $y$ are two points in $\Omega \backslash(B \cup K)$ that are separated by $K$ then they are also separated by $L$.

The expression "be separated by $K$ " means that $x$ and $y$ lie in different connected components of $\Omega \backslash K$.

Definition 3. A global minimizer in $\mathbb{R}^{N}$ is a pair $(u, K) \in \mathcal{A}$ (with $\Omega=\mathbb{R}^{N}$ ) such that for every ball $B$ in $\mathbb{R}^{N}$ and every competitor $(v, L)$ in $B$ we have

$$
\int_{B \backslash K}|\nabla u|^{2} d x+H^{N-1}(K \cap B) \leq \int_{B \backslash L}|\nabla v|^{2} d x+H^{N-1}(L \cap B)
$$

where $H^{N-1}$ denotes the Hausdorff measure of dimension $N-1$.

Proposition 9 on page 267 of [8] ensures that any blow up limit of a minimizer for the Mumford-Shah functional in $\mathbb{R}^{N}$, is a global minimizer in the sense of Definition 3. As a beginning for the description of global minimizers in $\mathbb{R}^{N}$, we can firstly think about what should be a global minimizer in $\mathbb{R}^{3}$. If $u$ is locally constant, then $K$ is a minimal cone, that is, a set that locally minimizes the Hausdorff measure of dimension 2 in $\mathbb{R}^{3}$. Then by [9] we know that $K$ is a cone of type $\mathrm{P}$ (hyperplane), Y (three half-planes meeting with 120 degrees angles) or of type $\mathbb{T}$ (cone over the edges of a regular tetraedron centered at the origin). Those cones became famous by the theorem of J. Taylor [20] which says that any minimal surface in $\mathbb{R}^{3}$ is locally $C^{1}$ equivalent to a cone of type $\mathbb{P}, \mathrm{Y}$ or ${ }^{\top} \mathrm{T}$.
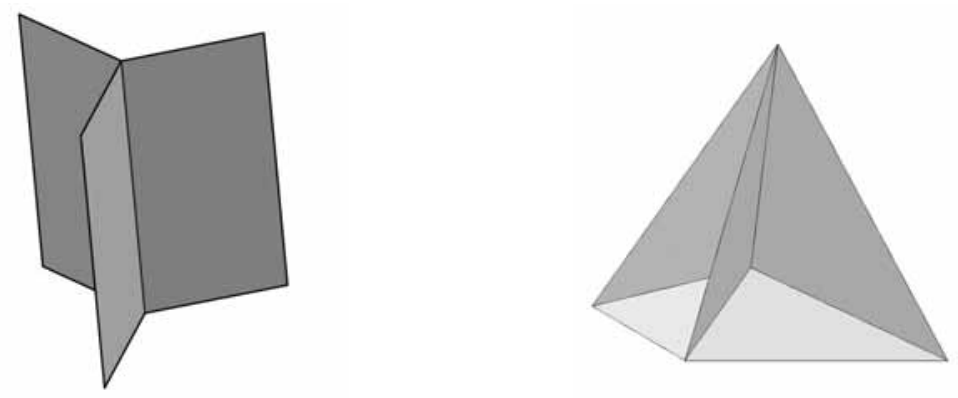

Fig. Cones of type $Y$ and $T$ in $\mathbb{R}^{3}$. 
To be clearer, this is a more precise definition of $Y$ and $T$, as in [10].

Definition 4. Define Prop $\subset \mathbb{R}^{2}$ by

$$
\begin{aligned}
& \text { Prop }=\left\{\left(x_{1}, x_{2}\right) ; x_{1} \geq 0, x_{2}=0\right\} \\
& \cup\left\{\left(x_{1}, x_{2}\right) ; x_{1} \leq 0, x_{2}=-\sqrt{3} x_{1}\right\} \\
& \cup\left\{\left(x_{1}, x_{2}\right) ; x_{1} \leq 0, x_{2}=\sqrt{3} x_{1}\right\} .
\end{aligned}
$$

Then let $Y_{0}=$ Prop $\times \mathbb{R} \subset \mathbb{R}^{3}$. The spine of $Y_{0}$ is the line $L_{0}=$ $=\left\{x_{1}=x_{2}=0\right\}$. A cone of type $Y$ is a set $Y=R\left(Y_{0}\right)$ where $R$ is the composition of a translation and a rotation. The spine of $Y$ is then the line $R\left(L_{0}\right)$.

Definition 5. Let $A_{1}=(1,0,0), \quad A_{2}=\left(-\frac{1}{3}, \frac{2 \sqrt{2}}{3}, 0\right), \quad A_{3}=$ $=\left(-\frac{1}{3},-\frac{\sqrt{2}}{3}, \frac{\sqrt{6}}{3}\right)$, and $A_{4}=\left(-\frac{1}{3},-\frac{\sqrt{2}}{3},-\frac{\sqrt{6}}{3}\right)$ the four vertices of $a$ regular tetrahedron centered at 0 . Let $T_{0}$ be the cone over the union of the 6 edges $\left[A_{i}, A_{j}\right] i \neq j$. The spine of $T_{0}$ is the union of the four halflines $\left[0, A_{j}[\right.$. A cone of type $T$ is a set $T=R\left(T_{0}\right)$ where $R$ is the composition of a translation and a rotation. The spine of $T$ is the image by $R$ of the spine of $T_{0}$.

So the pairs $(u, Z)$ where $u$ is locally constant and $Z$ is a minimal cone, are examples of global minimizers in $\mathbb{R}^{3}$. Another global minimizer can be obtained with $K_{\infty}$ a half-plane, by setting $u:=$ Craktip $\times \mathbb{R}$ (see [8] section 76). These examples are the only global minimizers in $\mathbb{R}^{3}$ that we know.

Note that if $(u, K)$ is a global minimizer in $\mathbb{R}^{N}$, then $u$ locally minimizes the Dirichlet integral in $\mathbb{R}^{N} \backslash K$. As a consequence, $u$ is harmonic in $\mathbb{R}^{N} \backslash K$. Moreover, if $B$ is a ball such that $K \cap B$ is regular enough, then the normal derivative of $u$ vanishes on $K \cap B$.

In this paper we wish to study global minimizers $(u, K)$ for which $K$ is a cone. It seems natural to think that any singular set of a global minimizer is a cone. But even if all known examples are cones, there is no proof of this fact. In addition, we will add some regularity on $K$. We denote by $S^{N-1}$ the unit sphere in $\mathbb{R}^{N}$ and, if $\Omega$ is a open set, $W^{1,2}(\Omega)$ is the Sobolev space. We will say that a domain $\Omega$ on $S^{N-1}$ has a piecewise $C^{2}$ boundary, if the topological boundary of $\Omega$, defined by $\partial \Omega=\bar{\Omega} \backslash \Omega$, consists of an union of $N-2$ dimensional hypersurfaces of class $C^{2}$. This allows some cracks, i.e. when $\Omega$ lies in each sides of its boundary. We will denote by $\tilde{\Sigma}$ the set of all the singular points of the boundary, that is

$$
\tilde{\Sigma}:=\left\{x \in \partial \Omega ; \forall r>0, B(x, r) \cap \partial \Omega \text { is not a } C^{2} \text { hypersurface }\right\} .
$$


Definition 6. A smooth cone is a set $K$ of dimension $N-1$ in $\mathbb{R}^{N}$ such that $K$ is conical, centered at the origin, and such that $S^{N-1} \backslash K$ is a domain with piecewise $C^{2}$ boundary. Moreover we assume that the embedding $W^{1,2}\left(S^{N-1} \backslash K\right) \rightarrow L^{2}\left(S^{N-1} \backslash K\right)$ is compact. Finally we suppose that we can strongly integrate by parts in $B(0,1) \backslash K$. More precisely, denoting by $\Sigma$ the set of singularities

$$
\Sigma:=\left\{t x ;(t, x) \in \mathbb{R}^{+} \times \tilde{\Sigma}\right\}
$$

we want that

$$
\int_{B(0,1) \backslash K}\langle\nabla u, \nabla \varphi\rangle=0
$$

for every harmonic function $u$ in $B(0,1) \backslash K$ with $\frac{\partial}{\partial n} u=0$ on $K \backslash \Sigma$, and for all $\varphi \in W^{1,2}(B(0,1) \backslash K)$ with vanishing trace on $S^{N-1} \backslash K$.

REMARK 7. For instance if $K$ is the cone over a finite union of $C^{2}$-arcs on $S^{2}$, then we can strongly integrate by parts in $B(0,1) \backslash K$. Another example in $\mathbb{R}^{N}$ is given by the union of admissible set of faces (as in Definition (22.2) of [5]).

Now this is the main result.

THEOREM 15. Let $(u, K)$ be a global minimizer in $\mathbb{R}^{N}$ and assume that $K$ is a smooth cone. Then there is $a \frac{1}{2}$-homogenous function $u_{1}$ such that $u-u_{1}$ is locally constant.

As we shall see, this result implies that if $(u, K)$ is a global minimizer in $\mathbb{R}^{N}$, and if $K$ is a smooth cone other than a minimal cone, then $\frac{3-2 N}{4}$ is an eigenvalue for the spherical Laplacian in $S^{N-1} \backslash K$ with Neumann boundary conditions. In section 2 we will give some applications about global minimizers in $\mathbb{R}^{3}$, using the estimates on the first eigenvalue that can be found in [6], [5] and [14]. More precisely, we have:

Proposition 17. Let $(u, K)$ be a global Mumford-Shah minimizer in $\mathbb{R}^{3}$ such that $K$ is a smooth cone. Moreover, assume that $S^{2} \cap K$ is a union of convex curvilinear polygons with $C^{\infty}$ sides. Then $u$ is locally constant and $K$ is a cone of type $\mathrm{P}, \mathrm{Y}$ or ${ }^{\mathrm{T}} \mathrm{T}$.

Another consequence of the main result is the following. 
Proposition 19. Let $(u, K)$ be a global Mumford-Shah minimizer in $\mathbb{R}^{3}$ such that $K$ is a half plane. Then $u$ is equal to a function of type cracktip $\times \mathbb{R}$, that is, in cylindrical coordinates,

$$
u(r, \theta, z)= \pm \sqrt{\frac{2}{\pi}} r^{\frac{1}{2}} \sin \frac{\theta}{2}+C
$$

for $0<r<+\infty,-\pi<\theta<\pi$ where $C$ is a constant.

Finally, we deduce two other consequences from Theorem 15. Let $(r, \theta, z) \in \mathbb{R}^{+} \times[-\pi, \pi] \times \mathbb{R}$ be the cylindrical coordinates in $\mathbb{R}^{3}$. For all $\omega \in[0, \pi]$ set

$$
\partial \Gamma_{\omega}:=\left\{(r, \theta, z) \in \mathbb{R}^{3} ; \theta=-\omega \text { or } \theta=\omega\right\} .
$$

and

$$
S_{\omega}:=\left\{(r, \theta, z) \in \mathbb{R}^{3} ; z=0, r>0, \theta \in[-\omega, \omega]\right\}
$$

Observe that $S_{0}$ is a half line, $S_{\frac{\pi}{2}}, \partial \Gamma_{0}$ and $\partial \Gamma_{\pi}$ are half-planes, and that $S_{\pi}$ and $\partial \Gamma_{\frac{\pi}{2}}$ are planes.

Proposition 18. There is no global Mumford-Shah minimizer in $\mathbb{R}^{3}$ such that $K$ is wing of type $\partial \Gamma_{\omega}$ with $\omega \notin\left\{0, \frac{\pi}{2}, \pi\right\}$.

Proposition 23. There is no global Mumford-Shah minimizer in $\mathbb{R}^{3}$ such that $K$ is an angular sector of type $\left(u, S_{\omega}\right)$ for $\omega \notin\left\{\frac{\pi}{2}, \pi\right\}$.

\section{If $K$ is a cone then $u$ is homogenous.}

In this section we want to prove Theorem 15 . Notice that this result is only useful if the dimension $N \geq 3$. Indeed, in dimension 2 , if $K$ is a cone then it is connected thus it is in the list described in the introduction.

\section{1 - Preliminary.}

Let us recall a standard uniqueness result about energy minimizers.

Proposition 8. Let $\Omega$ be an open and connected set of $\mathbb{R}^{N}$ and let $I \subset \partial \Omega$ be a hypersurface of class $C^{\infty}$. Suppose that $u$ and $v$ are two 
functions in $W^{1,2}(\Omega)$ such that $u=v$ a.e. on I (in terms of trace), solving the minimizing problem

$$
\min E(w):=\int_{\Omega}|\nabla w(x)|^{2} d x
$$

over all the functions $w \in W^{1,2}(\Omega)$ that are equal to $u$ and $v$ on $I$. Then

$$
u=v \text {. }
$$

Proof. This comes from a simple convexity argument which can be found for instance in [8], but let us write the proof since it is very short. By the parallelogram identity we have

$$
E\left(\frac{u+v}{2}\right)=\frac{1}{2} E(u)+\frac{1}{2} E(v)-\frac{1}{4} E(u-v) .
$$

On the other hand, since $\frac{u+v}{2}$ is equal to $u$ and $v$ on $I$, and by minimality of $u$ and $v$ we have

$$
E\left(\frac{u+v}{2}\right) \geq E(u)=E(v)
$$

Now by (2) we deduce that $E(u-v)=0$ and since $\Omega$ is connexe, this implies that $u-v$ is a constant. But $u-v$ is equal to 0 on $I$ thus $u=v$.

REMARK 9. The existence of a minimizer can also be proved using the convexity of $E(v)$.

\section{2 - Spectral decomposition.}

The key ingredient to obtain the main result will be the spectral theory of the Laplacian on the unit sphere. Since $u$ is harmonic, we will decompose $u$ as a sum of homogeneous harmonic functions just like we usually use the classical spherical harmonics. The difficulty here comes from the lack of regularity of $\mathbb{R}^{N} \backslash K$.

It will be convenient to work with connected sets. So let $\Omega$ be a connected component of $S^{N-1} \backslash K$, and let $A(r)$ be

$$
A(r):=\{t x ;(x, t) \in \Omega \times[0, r[\} .
$$

We also set

$$
A(\infty):=\left\{t x ;(x, t) \in \Omega \times \mathbb{R}^{+}\right\}
$$


All the following results are using that the embedding $W^{1,2}(\Omega)$ in $L^{2}(\Omega)$ is compact. Recall that this is the case by definition, since $K$ is a smooth cone. Notice that for instance the cone property insures that the embedding is compact (see Theorem 6.2. p. 144 of [1]).

Consider the quadratic form

$$
Q(u)=\int_{\Omega}|\nabla u(x)|^{2} d x
$$

of domain $W^{1,2}(\Omega)$ dense into the Hilbert space $L^{2}(\Omega)$. Since $Q$ is a positive and closed quadratic form (see for instance Proposition 10.61 p. 129 of [16]) there exists a unique selfadjoint operator denoted by $-\Delta_{n}$ of domain $D\left(-\Delta_{n}\right) \subset W^{1,2}(\Omega)$ such that

$$
\forall u \in D\left(-\Delta_{n}\right), \forall v \in W^{1,2}(\Omega), \quad \int_{\Omega}\langle\nabla u, \nabla v\rangle=\int_{\Omega}\left\langle-\Delta_{n} u, v\right\rangle .
$$

Proposition 10. The operator $-\Delta_{n}$ has a countably infinite discrete set of eigenvalues, whose eigenfunctions span $L^{2}(\Omega)$.

Proof. The proof is the same as if $\Omega$ was a regular domain. Consider the new quadratic form

$$
\tilde{Q}(u):=Q(u)+\|u\|_{2}^{2}
$$

with the same domain $W^{1,2}(\Omega)$. The form $\tilde{Q}$ has the same properties than $Q$ and the associated operator is Id $-\Delta_{n}$. Moreover $\tilde{Q}$ is coercive. As a result, the operator Id $-\Delta_{n}$ is bijective and its inverse goes from $L^{2}(\Omega)$ to $D\left(-\Delta_{n}\right) \subset W^{1,2}(\Omega)$. By hypothesis the embedding of $W^{1,2}(\Omega)$ into $L^{2}(\Omega)$ is compact. Thus the resolvant $\left(\mathrm{Id}-\Delta_{n}\right)^{-1}$ is a compact operator, and we conclude using the spectral theory of operators with a compact resolvant (see [19] Theorem XIII. 64 p. 245).

REMARK 11. The domain of $-\Delta_{n}$ is not known in general. If $\Omega$ was smooth, then we could show that the domain is exactly $D\left(-\Delta_{n}\right)=$ $=\left\{u \in W^{2,2}(\Omega) ; \frac{\partial u}{\partial n}=0\right.$ on $\left.\partial \Omega\right\}$. Here, the boundary of $\Omega$ has some singularities so this result doesn't apply directly. But knowing exactly the domain of $-\Delta_{n}$ will not be necessary for us.

Now we want to study the link between the abstract operator $\Delta_{n}$ and the classical spherical Laplacian $\Delta_{S}$ on the unit sphere. Recall that if we compute the Laplacian in spherical coordinates, we obtain the following 
equality

$$
\Delta=\frac{\partial^{2}}{\partial r}+\frac{N-1}{r} \frac{\partial}{\partial r}+\frac{1}{r^{2}} \Delta_{S}
$$

Proposition 12. For every function $f \in D\left(-\Delta_{n}\right)$ such that $-\Delta_{n} f=\lambda f$ we have

$$
\begin{aligned}
\text { i) } & f \in C^{\infty}(\Omega) \\
\text { ii) } & -\Delta_{S} f=-\Delta_{n} f=\lambda f \text { in } \Omega \\
\text { iii) } & \frac{\partial f}{\partial n} \text { exists and is equal to } 0 \text { on } K \cap \bar{\Omega} \backslash \Sigma
\end{aligned}
$$

Proof. Let $\varphi$ be a $C^{\infty}$ function with compact support in $\Omega$ and $f \in D\left(-\Delta_{n}\right)$. Then the Green formula in the distributional sense gives

$$
\int_{\Omega} \nabla f \cdot \nabla \varphi=\left\langle-\Delta_{S} f, \varphi\right\rangle
$$

where the left and right brackets mean the duality in the distributional sense. On the other hand, by definition of $-\Delta_{n}$ and since $f$ is in the domain $D\left(-\Delta_{n}\right)$, we also have

$$
\int_{\Omega} \nabla f . \nabla \varphi=\left\langle-\Delta_{n} f, \varphi\right\rangle
$$

where this time the brackets mean the scalar product in $L^{2}$. Therefore

$$
\Delta_{n} f=\Delta_{S} f \quad \text { in } \mathcal{D}^{\prime}(\Omega) .
$$

In other words, $-\Delta_{S} f=\lambda f$ in $\mathcal{D}^{\prime}(\Omega)$. But now since $f \in W^{1,2}(\Omega)$, by hypoellipticity of the Laplacian we know that $f$ is $C^{\infty}$ and that $-\Delta_{S} f=\lambda f$ in the classical sense. That proves $i$ ) and $i i$ ). We even know by the elliptic theory that, since $K \backslash \Sigma$ is regular, $f$ is regular at the boundary on $K \backslash \Sigma$.

Now consider a ball $B$ such that the intersection with $K \cap \bar{\Omega}$ does not meet $\Sigma$. Assume that $B$ is cut in two parts $B^{+}$and $B^{-}$by $K$, and that $B^{+}$is one part in $\Omega$. Possibly by modifying $B$ in a neighborhood of the intersection with $K$, we can assume that the boundary of $B^{+}$and $B^{-}$is $C^{2}$. The definition of $\Delta_{n}$ implies that for all function $\varphi \in C^{2}(\bar{\Omega})$ that vanishes out of $B^{+}$we have

$$
\int_{B^{+}}\langle\nabla f, \nabla \varphi\rangle d x=\int_{B^{+}}\left\langle-\Delta_{n} f, \varphi\right\rangle d x=\lambda \int_{B^{+}}\langle f, \varphi\rangle d x .
$$


On the other hand, integrating by parts,

$$
\begin{aligned}
\int_{B^{+}}\langle\nabla f, \nabla \varphi\rangle d x & =\int_{B^{+}}\left\langle-\Delta_{S} f, \varphi\right\rangle+\int_{\partial B^{+}} \frac{\partial u}{\partial n} \varphi \\
& =\lambda \int_{\partial B^{+}}\langle f, \varphi\rangle+\int_{\partial B^{+}} \frac{\partial f}{\partial n} \varphi
\end{aligned}
$$

thus

$$
\int_{\partial B^{+}} \frac{\partial f}{\partial n} \varphi=0 .
$$

In other words the function $f$ is a weak solution of the mixed boundary value problem

$$
\begin{aligned}
-\Delta_{S} u & =\lambda f \text { in } B^{+} \\
u & =f \text { on } \partial B^{+} \backslash K \\
\frac{\partial u}{\partial n} & =0 \text { on } K \cap \partial B^{+}
\end{aligned}
$$

Therefore, some results from the elliptic theory imply that $f$ is smooth in $B$ and is a strong solution (see [21]).

Let us recapitulate what we have obtained. For all function $f \in L^{2}(\Omega)$, there is a sequence of numbers $a_{i}$ such that

$$
f=\sum_{i=0}^{+\infty} a_{i} f_{i}
$$

where the sum converges in $L^{2}$. The functions $f_{i}$ are in $C^{\infty}(\Omega) \cap W^{1,2}(\Omega)$, verify $-\Delta_{S} f_{i}=\lambda_{i} f_{i}$ and $\frac{\partial f_{i}}{\partial n}=0$ on $K \cap \bar{\Omega} \backslash \Sigma$. Moreover, we can normalize the $f_{i}$ in order to obtain an orthonormal basis on $L^{2}(\Omega)$, in particular we have the following Parseval formula

$$
\|f\|_{2}^{2}=\sum_{i=0}^{+\infty}\left|a_{i}\right|^{2}
$$

Note that if $f$ belongs to the kernel of $-\Delta_{n}$ (i.e. is an eigenfunction with eigenvalue 0 ), then

$$
\langle\nabla f, \nabla f\rangle=\left\langle-\Delta_{n} f, f\right\rangle=0
$$


and since $\Omega$ is connected that means that $f$ is a constant. Thus 0 is the first eigenvalue and the associated eigenspace has dimension 1 . Then we can suppose that $\lambda_{0}=0$ and that all the $\lambda_{i}$ for $i>0$ are positive.

We define the scalar product in $W^{1,2}(\Omega)$ by

$$
\langle u, v\rangle_{W^{1,2}}:=\langle u, v\rangle_{L^{2}}+\langle\nabla u, \nabla v\rangle_{L^{2}} .
$$

Proposition 13. The family $\left\{f_{i}\right\}$ is orthogonal in $W^{1,2}(\Omega)$. Moreover if $f \in W^{1,2}(\Omega)$ and if its decomposition in $L^{2}(\Omega)$ is $f=\sum_{i=0}^{+\infty} a_{i} f_{i}$, then the $\operatorname{sum} \sum_{i=0}^{+\infty}\left|a_{i}\right|^{2} \|\left.\nabla f_{i}\right|_{2} ^{2}$ converges and

$$
\sum_{i=0}^{+\infty}\left|a_{i}\right|^{2}\left\|\nabla f_{i}\right\|_{2}^{2}=\|\nabla f\|_{2}^{2}
$$

Proof. We know that $\left\{f_{i}\right\}$ is an orthogonal family in $L^{2}(\Omega)$. In addition if $i \neq j$ then

$$
\begin{aligned}
\int_{\Omega} \nabla f_{i} \nabla f_{j} & =\int_{\Omega}-\Delta_{n} f_{i} f_{j} \\
& =\lambda_{i} \int_{\Omega} f_{i} f_{j} \\
& =0
\end{aligned}
$$

thus $\left\{f_{i}\right\}$ is also orthogonal in $W^{1,2}(\Omega)$ and

$$
\left\|f_{i}\right\|_{W^{1,2}}^{2}:=\left\|f_{i}\right\|_{2}^{2}+\left\|\nabla f_{i}\right\|_{2}^{2}=1+\lambda_{i} .
$$

Consider now the orthogonal projection (for the scalar product of $L^{2}$ )

$$
P_{k}: f \mapsto \sum_{i=0}^{k} a_{i} f_{i}
$$

The operator $P_{k}$ is the orthogonal projection on the closed subspace $A_{k}$ generated by $\left\{f_{0}, \ldots, f_{k}\right\}$. More precisely, we are interested in the restriction of $P_{k}$ to the subspace $W^{1,2}(\Omega) \subset L^{2}(\Omega)$. Also denote by $\tilde{P}_{k}: W^{1,2} \rightarrow A_{k}$ the orthogonal projection on the same subspace but for the scalar product of $W^{1,2}$. We want to show that $P_{k}=\tilde{P}_{k}$. To prove this, it suffice to show that for all sets of coefficients $\left\{a_{i}\right\}_{i=1 \ldots k}$ and $\left\{b_{i}\right\}_{i=1 \ldots k}$,

$$
\left\langle f-\sum_{i=0}^{k} a_{i} f_{i}, \sum_{i=0}^{k} b_{i} f_{i}\right\rangle_{W^{1,2}}=0 .
$$


Since we already have

$$
\left\langle f-\sum_{i=0}^{k} a_{i} f_{i}, \sum_{i=0}^{k} b_{i} f_{i}\right\rangle_{L^{2}}=0,
$$

all we have to show is that

$$
\int_{\Omega}\left\langle\nabla f-\sum_{i=0}^{k} a_{i} \nabla f_{i}, \sum_{i=0}^{k} b_{i} \nabla f_{i}\right\rangle d x=0 .
$$

Now

$$
\begin{aligned}
\int_{\Omega}\left\langle\nabla f-\sum_{i=0}^{k} a_{i} \nabla f_{i}, \sum_{i=0}^{k} b_{i} \nabla f_{i}\right\rangle & =\int_{\Omega}\left\langle\nabla f, \sum_{i=0}^{k} b_{i} \nabla f_{i}\right\rangle-\sum_{i=0}^{k} a_{i} b_{i}\left\|\nabla f_{i}\right\|_{2}^{2} \\
& =\sum_{i=0}^{k} b_{i}\left\langle-\Delta_{n} f_{i}, f\right\rangle_{L^{2}}-\sum_{i=0}^{k} a_{i} b_{i} \lambda_{i} \\
& =\sum_{i=0}^{k} a_{i} b_{i} \lambda_{i}-\sum_{i=0}^{k} a_{i} b_{i} \lambda_{i} \\
& =0
\end{aligned}
$$

thus $P_{k}=\tilde{P}_{k}$ and therefore, by Pythagoras

$$
\left\|P_{k}(f)\right\|_{W^{1,2}}^{2} \leq\|f\|_{W^{1,2}}^{2} .
$$

By letting $k$ tend to infinity we obtain

$$
\sum_{i=0}^{+\infty} a_{i}^{2}\left\|\nabla f_{i}\right\|_{2}^{2} \leq\|\nabla f\|_{2}^{2}
$$

From this inequality we deduce that the sum is absolutely converging in $W^{1,2}(\Omega)$. Therefore, the sequence of partial sum $\sum_{i=0}^{K} a_{i} f_{i}$ is a Cauchy sequence for the norm $W^{1,2}(\Omega)$. Thus, since the sum $\sum a_{i} f_{i}$ already converges to $f$ in $L^{2}(\Omega)$, by uniqueness of the limit the sum converges to $f$ in $W^{1,2}(\Omega)$, so we deduce that (6) is an equality and the proof is over.

Once we have a basis $\left\{f_{i}\right\}$ on $\Omega \subset S^{N-1}$, we consider for a certain $r_{0}>0$, the functions

$$
h_{i}(x)=r_{0}^{\alpha_{i}} f_{i}\left(\frac{x}{r_{0}}\right)
$$


defined on $r_{0} \Omega$. The exponent $\alpha_{i}$ is defined by

$$
\alpha_{i}=\frac{-(N-2)+\sqrt{(N-2)^{2}+4 \lambda_{i}}}{2} .
$$

The functions $h_{i}$ form a basis of $W^{1,2}\left(r_{0} \Omega\right)$. Indeed, if $f \in W^{1,2}\left(r_{0} \Omega\right)$, then $f\left(r_{0} x\right) \in W^{1,2}(\Omega)$ thus applying the decomposition on $\Omega$ we obtain

$$
f\left(r_{0} x\right)=\sum_{i=0}^{+\infty} b_{i} f_{i}(x)
$$

thus

$$
f(x)=\sum_{i=0}^{+\infty} a_{i} h_{i}(x)
$$

with

$$
a_{i}=b_{i} r_{0}^{-\alpha_{i}} .
$$

Notice that since $\left\|h_{i}\right\|_{2}^{2}=r_{0}^{2 \alpha_{i}+N-1}$ we also have

$$
\sum_{i=0}^{\infty} a_{i}^{2}\left\|h_{i}\right\|_{2}^{2}=\sum_{i=0}^{\infty} a_{i}^{2} r_{0}^{2 \alpha_{i}+N-1}=\|f\|_{L^{2}\left(r_{0} \Omega\right)}^{2}<+\infty
$$

Moreover, applying Proposition 13 we have that

$$
\sum_{i=0}^{\infty} b_{i}^{2}\left\|\nabla f_{i}\right\|_{2}^{2}=\left\|\nabla f\left(r_{0} x\right)\right\|_{2}^{2}<+\infty
$$

We are now able to state our decomposition in $A\left(r_{0}\right)$.

Proposition 14. Let $K$ be a smooth cone in $\mathbb{R}^{N}$, centered at the origin and let $\Omega$ be a connected component of $S^{N-1} \backslash K$. Then there exist some harmonic homogeneous functions $g_{i}$, orthogonal in $W^{1,2}(A(1))$, such that for every function $u \in W^{1,2}(A(1))$ harmonic in $A(1)$ with $\frac{\partial u}{\partial n}=0$ on $K \cap A(1) \backslash \Sigma$, and for every $\left.r_{0} \in\right] 0,1[$, we have that

$$
u=\sum_{i=0}^{+\infty} a_{i} g_{i} \quad \text { in } A\left(r_{0}\right)
$$

where the $a_{i}$ do not depend on radius $r_{0}$ and are unique. The sum converges in $W^{1,2}\left(A\left(r_{0}\right)\right)$ and uniformly on all compact sets of $A(1)$. Moreover

$$
\|u\|_{W^{1,2}\left(A\left(r_{0}\right)\right)}^{2}=\sum_{i=0}^{+\infty} a_{i}^{2}\left\|g_{i}\right\|_{W^{1,2}\left(A\left(r_{0}\right)\right)}^{2} .
$$


Proof. Since $u \in W^{1,2}(A(1))$ then for almost every $r_{0}$ in $\left.] 0,1\right]$ we have that

$$
\left.u\right|_{r_{0} \Omega} \in W^{1,2}\left(r_{0} \Omega\right) .
$$

Thus we can apply the decomposition on $r_{0} \Omega$ and say that

$$
u=\sum_{i=0}^{+\infty} a_{i} h_{i} \quad \text { on } r_{0} \Omega .
$$

Define $g_{i}$ by

$$
g_{i}(x):=\|x\|^{\alpha_{i}} f_{i}\left(\frac{x}{\|x\|}\right)
$$

where $\alpha_{i}$ is defined by (7). Since the $f_{i}$ are eigenfunctions for $-\Delta_{S}$, we deduce from (3) that

$$
\begin{aligned}
\Delta g_{i} & =\frac{\partial^{2}}{\partial r} g_{i}+\frac{N-1}{r} \frac{\partial}{\partial r} g_{i}+\frac{1}{r^{2}} \Delta_{S} g_{i} \\
& =\alpha_{i}\left(\alpha_{i}-1\right) r^{\alpha_{i}-2} f_{i}+\frac{N-1}{r} \alpha_{i} r^{\alpha_{i}-1} f_{i}-r^{\alpha_{i}-2} \lambda_{i} f_{i} \\
& =\left(\alpha_{i}^{2}+(N-2) \alpha_{i}-\lambda_{i}\right) r^{\alpha_{i}-2} f_{i} \\
& =0
\end{aligned}
$$

by definition of $\alpha_{i}$, thus the $g_{i}$ are harmonic in $A(+\infty)$. Notice that the $g_{i}$ are orthogonal in $L^{2}(A(1))$ because they are homogeneous and orthogonal in $L^{2}(\Omega)$. Note also that $h_{i}$ is equal to $g_{i}$ on $r_{0} \Omega$. Moreover for all $0<r \leq 1$ we have

$$
\begin{aligned}
\left\|g_{i}\right\|_{L^{2}(A(r))}^{2} & =\int_{A(r)}\left|g_{i}\right|^{2}=\int_{0}^{r} \int_{\partial B(t) \cap A(1)}\left|g_{i}(w)\right|^{2} d w d t \\
& =\int_{0}^{r} \int_{\Omega} t^{N-1}\left|g_{i}(t y)\right|^{2} d y d t=\int_{0}^{r} t^{2 \alpha_{i}+N-1} \int_{\Omega}\left|g_{i}(y)\right|^{2} d y d t \\
& =\frac{r^{2 \alpha_{i}+N}}{2 \alpha_{i}+N}\left\|f_{i}\right\|_{L^{2}(\Omega)}^{2}=\frac{r^{2 \alpha_{i}+N}}{2 \alpha_{i}+N} \leq 1 .
\end{aligned}
$$

On the other hand, since the $f_{i}$ and their tangential gradients are orthogonal in $L^{2}(\Omega)$, we deduce that the gradients of $g_{i}$ are orthogonal in $A(1)$. 
Then, by a computation similar to (12) we obtain for all $0<r \leq 1$

$$
\begin{aligned}
& \text { (13) }\left\|\nabla g_{i}\right\|_{L^{2}(A(r))}^{2}=\int_{0}^{r} \int_{\partial B(t) \cap A(1)}\left|\frac{\partial g_{i}}{\partial r}\right|^{2}+\left|\nabla_{\tau} g_{i}\right|^{2} d w d t \\
& =\int_{0}^{r} \int_{\partial B(t) \cap A(1)}\left|\alpha_{i} t^{\alpha_{i}-1} f_{i}\left(\frac{w}{t}\right)\right|^{2}+\left|t^{\alpha_{i}} \nabla_{\tau} f_{i}\left(\frac{w}{t}\right) \frac{1}{t}\right|^{2} d w d t \\
& =\alpha_{i}^{2} \int_{0}^{r} t^{2\left(\alpha_{i}-1\right)} \int_{\partial B(t) \cap A(1)}\left|f_{i}\left(\frac{w}{t}\right)\right|^{2} d w d t \\
& +\int_{0}^{r} t^{2\left(\alpha_{i}-1\right)} \int_{\partial B(t) \cap A(1)}\left|\nabla_{\tau} f_{i}\left(\frac{w}{t}\right)\right|^{2} d w d t \\
& =\alpha_{i}^{2} \int_{0}^{r} t^{2\left(\alpha_{i}-1\right)} \int_{\Omega}\left|f_{i}(w)\right|^{2} t^{N-1} d w d t \\
& +\int_{0}^{r} t^{2\left(\alpha_{i}-1\right)} \int_{\Omega}\left|\nabla_{\tau} f_{i}(w)\right|^{2} t^{N-1} d w d t \\
& =\alpha_{i}^{2} \frac{r^{2\left(\alpha_{i}-1\right)+N}}{2\left(\alpha_{i}-1\right)+N}\left\|f_{i}\right\|_{L^{2}(\Omega)}^{2}+\frac{r^{2\left(\alpha_{i}-1\right)+N}}{2\left(\alpha_{i}-1\right)+N}\left\|\nabla_{\tau} f_{i}\right\|_{L^{2}(\Omega)}^{2} \\
& =\frac{r^{2\left(\alpha_{i}-1\right)+N}}{2\left(\alpha_{i}-1\right)+N}\left(\alpha_{i}^{2}+\lambda_{i}\right)\left\|f_{i}\right\|_{L^{2}(\Omega)}^{2} \\
& \leq C r^{2 \alpha_{i}}\left(\alpha_{i}^{2}+\lambda_{i}\right)
\end{aligned}
$$

because $\left\|\nabla_{\tau} f_{i}\right\|_{2}^{2}=\lambda_{i}\left\|f_{i}\right\|_{2}^{2}, r \leq 1$ and $\alpha_{i} \geq 0$. Moreover the constant $C$ depends on the dimension $N$ but does not depend on $i$.

We denote by $g$ the function defined in $A(\infty)$ by

$$
g:=\sum_{i=0}^{+\infty} a_{i} g_{i}
$$

Then $g$ lies in $L^{2}\left(A\left(r_{0}\right)\right)$ because using (12) and (9)

$$
\|g\|_{L^{2}\left(A\left(r_{0}\right)\right)}^{2}=\sum_{i=0}^{+\infty}\left|a_{i}\right|^{2}\left\|g_{i}\right\|_{L^{2}\left(A\left(r_{0}\right)\right)}^{2} \leq \sum_{i=0}^{+\infty}\left|a_{i}\right|^{2} r_{0}^{2 \alpha_{i}+N}<+\infty .
$$

We want now to show that $g=u$. 
- First step: We claim that $g$ is harmonic in $A\left(r_{0}\right)$. Indeed, since the $g_{i}$ are all harmonic in $A\left(r_{0}\right)$, the sequence of partial sums $s_{k}:=\sum_{i=0}^{k} a_{i} g_{i}$ is a sequence of harmonic functions, uniformly bounded for the $L^{2}$ norm in each compact set of $A\left(r_{0}\right)$. By the Harnack inequality we deduce that the sequence of partial sums is uniformly bounded for the uniform norm in each compact set. Thus there is a subsequence that converges uniformly to a harmonic function, which in fact is equal to $g$ by uniqueness of the limit. Therefore, $g$ is harmonic in $A\left(r_{0}\right)$.

- Second step: We claim that $g$ belongs to $W^{1,2}\left(A\left(r_{0}\right)\right)$. Firstly, since $u \in W^{1,2}\left(r_{0} \Omega\right)$, by (8) and (10) we have that

$$
\sum_{i=0}^{+\infty} a_{i}^{2} r_{0}^{2 \alpha_{i}}\left\|\nabla_{\tau} f_{i}\right\|_{L^{2}(\partial B(0,1) \backslash K)}^{2}<+\infty .
$$

In addition, since $\left\|\nabla_{\tau} f_{i}\right\|_{2}^{2}=\lambda_{i}\left\|f_{i}\right\|_{2}^{2}$ and $\left\|f_{i}\right\|_{2}=1$, we deduce

$$
\sum_{i=0}^{+\infty} a_{i}^{2} r_{0}^{2 \alpha_{i}} \lambda_{i}<+\infty
$$

and since $\alpha_{i}$ and $\lambda_{i}$ are linked by the formula (7) we also have that

$$
\sum_{i=0}^{+\infty} a_{i}^{2} r_{0}^{2 \alpha_{i}} \alpha_{i}^{2}<+\infty
$$

Now, since $\sum a_{i} g_{i}$ converges absolutely on every compact set, we can say that

$$
\nabla g=\sum_{i=0}^{+\infty} a_{i} \nabla g_{i}
$$

thus using (13), (15), (16), and orthogonality,

$$
\begin{aligned}
\|\nabla g\|_{L^{2}\left(A\left(r_{0}\right)\right)}^{2} & =\sum_{i=0}^{+\infty} a_{i}^{2}\left\|\nabla g_{i}\right\|_{L^{2}}^{2} \\
& \leq C \sum_{i=0}^{+\infty} a_{i}^{2} r_{0}^{2 \alpha_{i}}\left(\alpha_{i}^{2}+\lambda_{i}\right)<+\infty .
\end{aligned}
$$

Therefore, $g \in W^{1,2}\left(A\left(r_{0}\right)\right)$.

- Third step : We claim that $\frac{\partial g}{\partial n}=0$ on $K \cap \overline{A\left(r_{0}\right)} \backslash \Sigma$. We already know that $\frac{\partial g_{i}}{\partial n}=0$ on $K \backslash \Sigma$ (because the $f_{i}$ have this property). We want to show 
that $g$ is so regular that we can exchange the order of $\frac{\partial}{\partial n}$ and $\sum$. So let $x_{0}$ be a point of $K \cap \overline{A\left(r_{0}\right)} \backslash \Sigma$ and let $B$ be a neighborhood of $x_{0}$ in $\mathbb{R}^{N}$ that doesn't meet $\Sigma$ and such that $K$ separates $B$ in two parts $B^{+}$and $B^{-}$. Assume that $B^{+}$is a part in $A\left(r_{0}\right)$. The sequence of partial sums $s_{k}:=\sum_{i=0}^{k} a_{i} g_{i}$ is a sequence of harmonic functions in $B^{+}$. Since $\partial B^{+} \cap K$ is $C^{2}$ we can do a reflection to extend $s_{k}$ in $B^{-}$. For all $k$, this new function $s_{k}$ is the solution of a certain elliptic equation whose operator become from the composition of the Laplacian with the application that makes $\partial B^{+} \cap K$ flat. Thus since $\sum a_{i} g_{i}$ converges absolutely for the $L^{2}$ norm, by the Harnack inequality $\sum a_{i} g_{i}$ converges absolutely for the uniform norm in a smaller neighborhood $B^{\prime} \subset B$ that still contains $x_{0}$. Thus $s_{k}$ converges to a $C^{1}$ function denoted by $s$, which is equal to $g$ on $B^{+}$. And since $\frac{\partial s_{k}}{\partial n}\left(x_{0}\right)=0$, by the absolute convergence of the sum we can exchange the order of the derivative and the symbol $\sum$ so we deduce that $\frac{\partial s}{\partial n}\left(x_{0}\right)=0$. Finally, since $s$ is equal to $g$ on $B^{+}$we deduce that $g$ is $C^{1}$ at the boundary and $\frac{\partial g}{\partial n}=0$ at $x_{0}$.

- Fourth step: we claim that $g$ is equal to $u$ on $r_{0} \Omega$. Let $r$ be a radius such that $r<r_{0}$. Then the function $x \mapsto g_{r}(x):=g\left(r \frac{x}{r_{0}}\right)$ is well defined for $x \in r_{0} \Omega$, and since the $g_{i}$ are homogeneous we have

$$
g\left(r \frac{x}{r_{0}}\right)=\sum_{i=0}^{+\infty} a_{i} g_{i}\left(r \frac{x}{r_{0}}\right)=\sum_{i=0}^{+\infty}\left(\frac{r}{r_{0}}\right)^{\alpha_{i}} a_{i} g_{i}(x)=\sum_{i=0}^{+\infty}\left(\frac{r}{r_{0}}\right)^{\alpha_{i}} a_{i} h_{i}(x)
$$

We deduce that the function $x \mapsto g\left(\frac{r}{r_{0}} x\right)$ is in $L^{2}\left(r_{0} \Omega\right)$ and its coefficients in the basis $\left\{h_{i}\right\}$ are $\left\{\left(\frac{r}{r_{0}}\right)^{\alpha_{i}} a_{i}\right\}$. We want to show that $\left\|g_{r}-u\right\|_{L^{2}\left(r_{0} \Omega\right)}$ tend to 0 . Indeed, writing $u$ in the basis $\left\{h_{i}\right\}$

$$
u=\sum_{i=0}^{+\infty} a_{i} h_{i}
$$

we obtain

$$
\left\|g_{r}-u\right\|_{2}^{2}=\sum_{i=0}^{+\infty}\left(\left(\frac{r}{r_{0}}\right)^{\alpha_{i}}-1\right)^{2} a_{i}^{2}\left\|h_{i}\right\|_{2}^{2}
$$


which tends to zero when $r$ tends to $r_{0}$ by the dominated convergence theorem because $\left(\left(\frac{r}{r_{0}}\right)^{\alpha_{i}}-1\right)^{2} \leq 1$. Therefore, there is a subsequence for which $g_{r}$ tends to $u$ almost everywhere. On the other hand, since $g$ is harmonic, the limit of $g_{r}$ exists and is equal to $g$. That means that $g$ tends to $u$ radially at almost every point of $r_{0} \Omega$.

- Fifth step: The functions $u$ and $g$ are harmonic functions in $A\left(r_{0}\right)$, with finite energy, with a normal derivative equal to zero on $K \cap \overline{A\left(r_{0}\right)} \backslash \Sigma$ and that coïncide on $\partial A\left(r_{0}\right) \backslash K$. To show that $u=g$ in $A\left(r_{0}\right)$ we shall prove that $g$ is an energy minimizer. Proposition 8 will then give the uniqueness.

Let $\left.\varphi \in W^{1,2}\left(A\left(r_{0}\right)\right) \backslash K\right)$ have a vanishing trace on $\partial B\left(0, r_{0}\right)$. Then, setting $J(v):=\int_{A\left(r_{0}\right)}|\nabla v|^{2}$ for $v \in W^{1,2}\left(A\left(r_{0}\right)\right)$ we have

$$
J(g+\varphi)=J(g)+\int_{A\left(r_{0}\right)} \nabla g \nabla \varphi+J(\varphi) .
$$

Now since $g$ is harmonic with Neumann condition on $K \backslash \Sigma$ and since $\varphi$ vanishes on $r_{0} \Omega$, integrating by parts we obtain

$$
J(g+\varphi)=J(g)+J(\varphi) .
$$

Since $J$ is non negative and $g+\varphi$ describes all the functions in $W^{1,2}\left(A\left(r_{0}\right)\right)$ with trace equal to $u$ on $r_{0} \Omega$, we deduce that $g$ minimizes $J$. We can do the same with $u$ thus $u$ and $g$ are two energy minimizers with same boundary conditions. Therefore, by Proposition 8 we know that $g=u$.

- Sixth step: The decomposition do not depends on $r_{0}$. Indeed, let $r_{1}$ be a second choice of radius. Then we can do the same work as before to obtain a decomposition

$$
u(x):=\sum_{i=0}^{+\infty} b_{i} g_{i}(x) \quad \text { in } B\left(0, r_{1}\right) \backslash K .
$$

Now by uniqueness of the decomposition in $B\left(0, \min \left(r_{0}, r_{1}\right)\right)$ we deduce that $b_{i}=a_{i}$ for all $i$.

In addition, $r_{0}$ was initially chosen almost everywhere in $] 0,1[$. But since the decomposition does not depend on the choice of radius, $r_{0}$ can be chosen anywhere in $] 0,1[$, by choosing a radius almost everywhere in $] r_{0}, 1[$.

THEOREM 15. Let $(u, K)$ be a global minimizer in $\mathbb{R}^{N}$ such that $K$ is a smooth cone. Then for each connected component of $\mathbb{R}^{N} \backslash K$ there is a constant $u_{k}$ such that $u-u_{k}$ is $\frac{1}{2}$-homogenous. 
Proof. Let $\Omega$ be a connected component of $\mathbb{R}^{N} \backslash K$. We apply the preceding proposition to $u$. Thus

$$
u(x)=\sum_{i=0}^{+\infty} a_{i} g_{i}(x) \quad \text { in } A\left(r_{0}\right) .
$$

for a certain radius $r_{0}$ chosen in $] 0,1[$. Let us prove that the same decomposition is true in $A(\infty)$. Applying Proposition 14 to the function $u_{R}(x)=u(R x)$ we know that there are some coefficients $a_{i}(R)$ such that

$$
u_{R}(x)=\sum_{i=0}^{+\infty} a_{i}(R) g_{i}(x) \text { in } A\left(r_{0}\right) .
$$

Now since $u_{R}\left(\frac{x}{R}\right)=u(x)$ we can use the homogeneity of the $g_{i}$ to identify the terms in $B\left(0, r_{0}\right)$ thus $a_{i}(R)=a_{i} R^{\alpha_{i}}$. Now we fix $y=R x$ and we obtain that

$$
u(y)=\sum_{i=0}^{+\infty} a_{i} g_{i}(y) \text { in } A\left(R r_{0}\right) .
$$

Since $R$ is arbitrary the decomposition is true in $A(\infty)$.

In addition for every radius $R$ we know that

$$
\|\nabla u\|_{L^{2}(A(R))}^{2}=\sum_{i=0}^{+\infty} a_{i}^{2}\left\|\nabla g_{i}\right\|_{L^{2}(A(R))}^{2}
$$

and since $g_{i}$ is $\alpha_{i}$-homogenous,

$$
\left\|\nabla g_{i}\right\|_{L^{2}(A(R))}^{2}=R^{2\left(\alpha_{i}-1\right)+N}\left\|\nabla g_{i}\right\|_{L^{2}(A(1))}^{2} .
$$

Now, since $u$ is a global minimizer, a classical estimate on the gradient obtained by comparing $(u, K)$ with $(v, L)$ where $v=\mathbf{1}_{B(0, R)^{c}} u$ and $L=\partial B(0, R) \cup(K \backslash B(0, R))$ gives that there is a constant $C$ such that for all radius $R$

$$
\|\nabla u\|_{L^{2}(B(0, R) \backslash K)}^{2} \leq C R^{N-1} .
$$

We deduce

$$
\sum_{i=0}^{+\infty} a_{i}^{2} R^{2\left(\alpha_{i}-1\right)+N}\left\|\nabla g_{i}\right\|_{L^{2}(A(1))}^{2} \leq C R^{N-1} .
$$

Thus

$$
\sum_{i=0}^{+\infty} a_{i}^{2} R^{2 \alpha_{i}-1}\left\|\nabla g_{i}\right\|_{L^{2}(A(1))}^{2} \leq C
$$


This last quantity is bounded when $R$ goes to infinity if and only if $a_{i}=0$ whenever $\alpha_{i}>1 / 2$. On the other hand, this quantity is bounded when $R$ goes to 0 , if and only if $a_{i}=0$ whenever $0<\alpha_{i}<1 / 2$. Therefore, $u-a_{0}$ is a finite sum of terms of degree $\frac{1}{2}$.

REMARK 16. In Chapter 65 of [8], we can find a variational argument that leads to a formula in dimension 2 that links the radial and tangential derivatives of $u$. For all $\xi \in K \cap \partial B(0, r)$, we call $\theta_{\xi} \in\left[0, \frac{\pi}{2}\right]$ the non oriented angle between the tangent to $K$ at point $\xi$ and the radius $[0, \xi]$. Then we have the following formula

$$
\int_{\partial B(0, r) \backslash K}\left(\frac{\partial u}{\partial r}\right)^{2} d H^{1}=\int_{\partial B(0, r) \backslash K}\left(\frac{\partial u}{\partial \tau}\right)^{2} d H^{1}+\sum_{\xi \in K \cap \partial B(0, r)} \cos \theta_{\xi}-\frac{1}{r} H^{1}(K \cap B(0, r)) .
$$

Notice that for a global minimizer in $\mathbb{R}^{2}$ with $K$ a centered cone we find

$$
\int_{\partial B(0, r) \backslash K}\left(\frac{\partial u}{\partial r}\right)^{2} d H^{1}=\int_{\partial B(0, r) \backslash K}\left(\frac{\partial u}{\partial \tau}\right)^{2} d H^{1} .
$$

Now suppose that $(u, K)$ is a global minimizer in $\mathbb{R}^{N}$ with $K$ a smooth cone centered at 0 . Then by Theorem 15 we know that $u$ is harmonic and $\frac{1}{2}$-homogenous. Its restriction to the unit sphere is an eigenfunction for the spherical Laplacian with Neumann boundary condition and associated to the eigenvalue $\frac{2 N-3}{4}$. We deduce that

$$
\left\|\nabla_{\tau} u\right\|_{L^{2}(\partial B(0,1))}^{2}=\frac{2 N-3}{4}\|u\|_{L^{2}(\partial B(0,1))}^{2} .
$$

On the other hand

$$
\frac{\partial u}{\partial r}(x)=\frac{1}{2}\|x\|^{-\frac{1}{2}} u\left(\frac{x}{\|x\|}\right)
$$

thus

$$
\left\|\frac{\partial u}{\partial r}\right\|_{L^{2}(\partial B(0,1))}^{2}=\frac{1}{4}\|u\|_{L^{2}(\partial B(0,1))}^{2} .
$$

So

$$
\left\|\nabla_{\tau} u\right\|_{L^{2}(\partial B(0,1))}^{2}=(2 N-3)\left\|\frac{\partial u}{\partial r}\right\|_{L^{2}(\partial B(0,1))}^{2} .
$$

In particular, for $N=2$ we have the same formula as (18). 


\section{Some applications.}

As it was claimed in the introduction, here is some few applications of Theorem 15.

Proposition 17. Let $(u, K)$ be a global minimizer in $\mathbb{R}^{3}$ such that $K$ is a smooth cone. Moreover, assume that $S^{2} \cap K$ is a union of convex curvilinear polygons with $C^{\infty}$ sides. Then $u$ is locally constant and $K$ is a cone of type $\mathrm{P}, \mathrm{Y}$ or $\mathrm{T}$.

Proof. In each polygon we know by Proposition 4.5. of [6] that the smallest positive eigenvalue for the operator minus Laplacian with Neumann boundary conditions is greater than or equal to 1 . Thus it cannot be $\frac{3}{4}$ and $u$ is locally constant. Then $K$ is a minimal cone in $\mathbb{R}^{3}$ and we know from [9] that it is a cone of type $\mathbb{P}, \mathbb{Y}$ or $T$.

Let $(r, \theta, z) \in \mathbb{R}^{+} \times[-\pi, \pi] \times \mathbb{R}$ be the cylindrical coordinates in $\mathbb{R}^{3}$. For every $\omega \in[0, \pi]$ set

$$
\Gamma_{\omega}:=\left\{(r, \theta, z) \in \mathbb{R}^{3} ;-\omega<\theta<\omega\right\}
$$

of boundary

$$
\partial \Gamma_{\omega}:=\left\{(r, \theta, z) \in \mathbb{R}^{3} ; \theta=-\omega \text { or } \theta=\omega\right\} .
$$

Consider $\Omega_{\omega}=\Gamma_{\omega} \cap S^{2}$ and let $\lambda_{1}$ be the smallest positive eigenvalue of $-\Delta_{S}$ in $\Omega_{\omega}$ with Neumann conditions on $\partial \Omega_{\omega}$. Then by Lemma 4.1. of [6] we have that

$$
\lambda_{1}=\min \left(2, \lambda_{\omega}\right)
$$

where

$$
\lambda_{\omega}=\left(\frac{\pi}{2 \omega}+\frac{1}{2}\right)^{2}-\frac{1}{4}
$$

In particular for the cone of type $\mathrm{Y}, \omega=\frac{\pi}{3}$ thus $\lambda_{1}=2$.

Observe that for $\omega \neq \pi, \lambda_{\omega} \neq \frac{3}{4}$. So we get this following proposition.

Proposition 18. There is no global Mumford-Shah minimizer in $\mathbb{R}^{3}$ such that $K$ is wing of type $\partial \Gamma_{\omega}$ with $\omega \notin\left\{0, \frac{\pi}{2}, \pi\right\}$. 
Another consequence of Theorem 15 is the following. Let $P$ be the half plane

$$
P:=\left\{(r, \theta, z) \in \mathbb{R}^{3} ; \theta=\pi\right\} .
$$

Proposition 19. Let $(u, K)$ be a global Mumford-Shah minimizer in $\mathbb{R}^{3}$ such that $K=P$. Then $u$ is equal to cracktip $\times \mathbb{R}$, that is in cylindrical coordinates

$$
u(r, \theta, z)= \pm \sqrt{\frac{2}{\pi}} r^{\frac{1}{2}} \sin \frac{\theta}{2}+C
$$

for $0<r<+\infty$ and $-\pi<\theta<\pi$.

REMARK 20. In Section 3 we will give a second proof of Proposition 19.

REMARK 21. We already know that $u=$ cracktip $\times \mathbb{R}$ is a global minimizer in $\mathbb{R}^{3}$ (see [8]).

To prove Proposition 19 we will use the following well known result.

Proposition 22 ([5], [13]). The smallest positive eigenvalue for $-\Delta_{n}$ in $S^{2} \backslash P$ is $\frac{3}{4}$, the corresponding eigenspace is of dimension 1 generated by the restriction on $S^{2}$ of the following function in cylindrical coordinates

$$
u(r, \theta, z)=r^{\frac{1}{2}} \sin \frac{\theta}{2}
$$

for $0<r<+\infty$ and $-\pi<\theta<\pi$.

Now the proof of Proposition 19 can be easily deduce from Proposition 22 and Theorem 15.

Proof of Proposition 19. If $(u, P)$ is a global minimizer, we know that after removing a constant the restriction of $u$ to the unit sphere is an eigenfunction for $-\Delta_{n}$ in $S^{2} \backslash P$ associated to the eigenvalue $\frac{3}{4}$. Therefore, from Proposition 22 we know that

$$
u(r, \theta, z)=C r^{\frac{1}{2}} \sin \frac{\theta}{2}
$$

so we just have to determinate the constant $C$. But by a well known argument about Mumford-Shah minimizers we prove that $C$ must be equal to $\pm \sqrt{\frac{2}{\pi}}$ (see [8] Section 61 for more details). 
Now set

$$
S_{\omega}:=\{(r, \theta, 0) ; r>0, \theta \in[-\omega, \omega]\}
$$

Proposition 23. There is no global Mumford-Shah minimizer in $\mathbb{R}^{3}$ such that $K$ is an angular sector of type $\left(u, S_{\omega}\right)$ for $0<\omega<\frac{\pi}{2}$ or $\frac{\pi}{2}<\omega<\pi$.

Proof. According to Theorem 15, if $\left(u, S_{\omega}\right)$ is a global minimizer, then $u-u_{0}$ is a homogenous harmonic function of degree $\frac{1}{2}$, thus its restriction to $S^{2} \backslash S_{\omega}$ is an eigenfunction for $-\Delta_{n}$ associated to the eigenvalue $\frac{3}{4}$. Now if $\lambda(\omega)$ denotes the smallest eigenvalue on $\partial B(0,1) \backslash S_{\omega}$, we know by Theorem 2.3.2. p. 47 of [14] that $\lambda(\omega)$ is non decreasing with respect to $\omega$. Since $\lambda\left(\frac{\pi}{2}\right)=\frac{3}{4}$, we deduce that for $\omega<\frac{\pi}{2}$, we have

$$
\lambda(\omega) \geq \frac{3}{4} .
$$

In [14] page 53 we can find the following asymptotic formula near $\omega=\frac{\pi}{2}$

$$
\lambda(\omega)=\frac{3}{4}+\frac{2}{\pi} \cos \omega+O\left(\cos ^{2} \omega\right) .
$$

this proves that the case when (19) is a equality only arises when $\omega=\frac{\pi}{2}$. Thus such eigenfunction $u$ doesn't exist.

Consider now the case $\omega>\frac{\pi}{2}$. For $\omega=\pi$ there are tow connected components. Thus 0 is an eigenvalue of multiplicity 2 . The second eigenvalue is equal to 2 . Therefore, for $\omega=\pi$ the spectrum is

$$
0 \leq 0 \leq 2 \leq \lambda_{3} \leq \ldots \quad \omega=\pi
$$

By monotonicity, when $\omega$ decreases, the eigenvalues increase. Since the domain becomes connexe, 0 become of multiplicity 1 thus the second eigenvalue become positive. The spectrum is now

$$
0 \leq \lambda_{1} \leq \lambda_{2} \leq \ldots \quad \omega<\pi
$$

with $\lambda_{i} \geq 2$ for $i \geq 2$. Thus the only eigenvalue that could be equal to $\frac{3}{4}$ is $\lambda_{2}$ which is increasing from from 0 to $\frac{3}{4}$, reached for $\omega=\frac{\pi}{2}$. Now (20) says that the increasing is strict near $\omega=\frac{\pi}{2}$. Therefore there is no eigenvalue equal to $3 / 4$ for $\omega>\frac{\pi}{2}$ and there is no possible global minimizer. 


\section{Second proof of Propositions 19 and 22.}

Here we want to give a second proof of Proposition 19, without using Theorem 15, and which do not use Proposition 22. In a remark at the end of this section, we will briefly explain how to use this proof of Proposition 19 in order to obtain a new proof of Proposition 22 as well.

Let assume that $K$ is a half plane in $\mathbb{R}^{3}$. We can suppose for instance that

$$
K=P:=\left\{x_{2}=0\right\} \cap\left\{x_{1} \leq 0\right\}
$$

We begin by studying the harmonic measure in $\mathbb{R}^{3} \backslash P$.

Let $B:=B(0, R)$ be a ball of radius $R$ and let $\gamma$ be the trace operator on $\partial B(0, R) \backslash P$. We denote by $T$ the image of $W^{1,2}(B \backslash K)$ by $\gamma$. We also denote by $C_{b}^{0}(\partial B \backslash K)$ the set of continuous and bounded functions on $\partial B(0,1) \backslash P$. Finally set $A:=T \cap C_{b}^{0}$. Obviously $A$ is not empty. To every function $f \in A$, Proposition 15.6. of [8] associates a unique energy minimizing function $u \in W^{1,2}(B \backslash K)$ such that $\gamma(u)=f$ on $\partial B \backslash P$. Since $u$ is harmonic we know that it is $C^{\infty}$ in $B \backslash K$. Let $y \in B \backslash K$ be a fixed point and consider the linear form $\mu_{y}$ defined by

$$
\begin{aligned}
\mu_{y}: A & \rightarrow \mathbb{R} \\
f & \mapsto u(y) .
\end{aligned}
$$

By the maximum principle for energy minimizers, we know that for all $f \in A$ we have

$$
\left|\mu_{y}(f)\right| \leq\|f\|_{\infty}
$$

thus $\mu_{y}$ is a continuous linear form on $A$ for the norm \|\|$_{\infty}$. We identify $\mu_{y}$ with its representant in the dual space of $A$ and we call it harmonic measure.

Moreover, the harmonic measure is positive. That is, if $f \in A$ is a non negative function, then (by the maximum principle) $\mu_{y}(f)$ is non negative. By positivity of $\mu_{y}$, if $f \in A$ is a non negative function and $g \in A$ is such that $f g \in A$, then since $\left(\|g\|_{\infty}+g\right) f$ and $\left(\|g\|_{\infty}-g\right) f$ are two non negative functions of $A$ we deduce that

$$
\left|\left\langle f g, \mu_{y}\right\rangle\right| \leq\|g\|_{\infty}\left\langle f, \mu_{y}\right\rangle .
$$

Now here is an estimate on the measure $\mu_{y}^{R}$. 
Lemma 24. There is a dimensional constant $C_{N}$ such that the following holds. Let $R$ be a positive radius. For $0<\lambda<\frac{R}{2}$ consider the spherical domain

$$
\mathcal{C}_{\lambda}:=\left\{x \in \mathbb{R}^{3} ;|x|=R \text { and } d(x, P) \leq \lambda\right\} .
$$

Let $\varphi_{\lambda} \in C^{\infty}(\partial B(0, R))$ be a function between 0 and 1 , that is equal to 1 on $\mathcal{C}_{\lambda}$ and 0 on $\partial B(0, R) \backslash \mathcal{C}_{2 \lambda}$ and that is symmetrical with respect to $P$. Then for every $y \in B\left(0, \frac{R}{2}\right) \backslash P$ we have

$$
\mu_{y}^{R}\left(\varphi_{\lambda}\right) \leq C \frac{\lambda}{R}
$$

Proof. Since $\varphi_{\lambda}$ is continuous and symmetrical with respect to $P$, by the reflection principle, its harmonic extension $\varphi$ in $B(0, R)$ has a normal derivative equal to zero on $P$ in the interior of $B(0, R)$. Moreover $\varphi_{\lambda}$ is clearly in the space $A$. Thus by definition of $\mu_{y}$,

$$
\varphi(y)=\left\langle\varphi_{\lambda}, \mu_{y}^{R}\right\rangle .
$$

On the other hand, since $\varphi_{\lambda}$ is continuous on the entire sphere, we also have the formula with the classical Poisson kernel

$$
\varphi(y)=\frac{R^{2}-|y|^{2}}{N \omega_{N} R} \int_{\partial B_{R}} \frac{\varphi_{\lambda}(x)}{|x-y|^{3}} d s(x)
$$

with $\omega_{N}$ equal to the measure of the unit sphere. In other words

$$
\mu_{y}^{R}\left(\varphi_{\lambda}\right)=\frac{R^{2}-|y|^{2}}{N \omega_{N} R} \int_{\partial B_{R}} \frac{\varphi_{\lambda}(x)}{|x-y|^{3}} d s(x) .
$$

For $x \in \partial B_{R}$ we have

$$
\frac{1}{2} R \leq|x|-|y| \leq|x-y| \leq|x|+|y| \leq \frac{3}{2} R .
$$

We deduce that

$$
\mu_{y}^{R}\left(\varphi_{\lambda}\right) \leq C_{N} \frac{1}{R^{2}} \int_{\mathcal{C}_{2 \lambda}} d s
$$


Now integrating by parts,

$$
\begin{aligned}
\int_{\mathcal{C}_{\lambda}} d s & =2 \int_{0}^{\lambda} 2 \pi \sqrt{R^{2}-w^{2}} d w \\
& =4 \pi \frac{\lambda}{2} \sqrt{R^{2}-\lambda^{2}}+R^{2} \arcsin \left(\frac{\lambda}{R}\right) \\
& \leq C R \lambda
\end{aligned}
$$

because $\arcsin (x) \leq \frac{\pi}{2} x$. The proposition follows.

Now we can prove the uniqueness of cracktip $\times \mathbb{R}$.

Second Proof of Proposition 19. Let us show that $u$ is vertically constant. Let $t$ be a positive real. For $x=\left(x_{1}, x_{2}, x_{3}\right) \in \mathbb{R}^{3}$ set $x_{t}:=\left(x_{1}, x_{2}, x_{3}+t\right)$. We also set

$$
u_{t}(x):=u(x)-u\left(x_{t}\right)
$$

Since $u$ is a function associated to a global minimizer, and since $K$ is regular, we know that for all $R>0$, the restriction of $u$ to the sphere $\partial B(0, R) \backslash K$ is continuous and bounded on $\partial B(0, R) \backslash K$ with finite limits on each sides of $K$. It is the same for $u_{t}$. Thus for all $x \in \mathbb{R}^{3} \backslash P$ and for all $R>2\|x\|$ we can write

$$
u_{t}(x):=\left\langle\left. u_{t}\right|_{\partial B(0, R) \backslash P}, \mu_{x}^{R}\right\rangle
$$

where $\mu_{x}$ is the harmonic measure defined in (22). We want to prove that for $x \in \mathbb{R}^{3} \backslash P,\left\langle\left. u_{t}\right|_{\partial B(0, R) \backslash P}, \mu_{x}^{R}\right\rangle$ tends to 0 when $R$ goes to infinity. This will prove that $u_{t}=0$.

So let $x \in \mathbb{R}^{3} \backslash P$ be fixed. We can suppose that $R>100(\|x\|+t)$. Let $\mathcal{C}_{\lambda}$ and $\varphi_{\lambda}$ be as in Lemma 24 . Then write

$$
u_{t}(x)=\left\langle\left. u_{t}\right|_{\partial B(0, R) \backslash P} \varphi_{\lambda}, \mu_{x}^{R}\right\rangle+\left\langle\left. u_{t}\right|_{\partial B(0, R) \backslash P}\left(1-\varphi_{\lambda}\right), \mu_{x}^{R}\right\rangle .
$$

Now by a standard estimate on Mumford-Shah minimizers (that comes from Campanato's Theorem, see [3] p. 371) we have for all $x \in \mathbb{R}^{N} \backslash P$,

$$
\left|u_{t}(x)\right| \leq C \sqrt{t}
$$

Then, using Lemma 24 we obtain

$$
\left|\left\langle\left. u_{t}\right|_{\partial B(0, R) \backslash P} \varphi_{\lambda}, \mu_{x}^{R}\right\rangle\right| \leq C \sqrt{t} \frac{\lambda}{R} .
$$


On the other hand, for the points $y$ such that $d(y, P) \geq \lambda$, since $\tilde{u}: u()-.u(y)$ is harmonic in $B(y, d(y, P))$ we have, by a classical estimation on harmonic functions (see the introduction of [12])

$$
|\nabla \tilde{u}(y)| \leq C \frac{1}{d(y, P)}\|\tilde{u}\|_{L^{\infty}\left(\partial B\left(y, \frac{1}{2} d(y, P)\right)\right)} .
$$

Now using Campanato's Theorem again we know that

$$
\|\tilde{u}\|_{L^{\infty}\left(\partial B\left(y, \frac{1}{2} d(y, P)\right)\right)} \leq C d(y, P)^{\frac{1}{2}}
$$

thus

$$
|\nabla u(y)| \leq C \frac{1}{d(y, P)^{\frac{1}{2}}}
$$

and finally by the mean value theorem we deduce that for all the points $y$ such that $d(y, P) \geq \lambda$,

$$
\left|u_{t}(y)\right| \leq C \sup _{z \in\left[y, y_{t}\right]}|\nabla u(z)| \cdot\left|y-y_{t}\right| \leq t \frac{1}{\lambda^{\frac{1}{2}}} .
$$

Therefore,

$$
\left|\left\langle\left. u_{t}\right|_{\partial B(0, R) \backslash P}\left(1-\varphi_{\lambda}\right), \mu_{x}^{R}\right\rangle\right| \leq C t \frac{1}{\lambda^{\frac{1}{2}}} .
$$

So

$$
\left|u_{t}(x)\right| \leq C \sqrt{t} \frac{\lambda}{R}+C t \frac{1}{\lambda^{\frac{1}{2}}}
$$

thus by setting $\lambda=R^{\frac{1}{2}}$ and by letting $R$ go to $+\infty$ we deduce that $u_{t}(x)=0$ thus $z \mapsto u(x, y, z)$ is constant.

Now we fix $z_{0}=0$ and we introduce $P_{0}:=P \cap\{z=0\}$. We want to show that $\left(u(x, y, 0), P_{0}\right)$ is a global minimizer in $\mathbb{R}^{2}$. Let $(v(x, y), \Gamma)$ be a competitor for $u(x, y, 0)$ in the 2 -dimensional ball $B$ of radius $\rho$. Let $\mathcal{C}$ be the cylinder $\mathcal{C}:=B \times[-R, R]$. Define $\tilde{v}$ and $\tilde{\Gamma}$ in $\mathbb{R}^{3}$ by

$$
\begin{gathered}
\tilde{v}(x, y, z)=\left\{\begin{array}{cc}
v(x, y) & \text { if }(x, y, z) \in \mathcal{C} \\
u(x, y, z) & \text { if }(x, y, z) \notin \mathcal{C}
\end{array}\right. \\
\tilde{\Gamma}:=(\mathcal{C} \cap[\Gamma \times[-R, R]]) \cup(P \backslash \mathcal{C}) \cup(B \times\{ \pm R\}) .
\end{gathered}
$$

It is a topological competitor because $\mathbb{R}^{3} \backslash P$ is connected (thus $P$ doesn't separate any points). Now finally let $\tilde{B}$ be a ball that contains $\mathcal{C}$. Then $(\tilde{v}, \tilde{\Gamma})$ 
is a competitor for $(u, P)$ in $\tilde{B}$. By minimality we have:

$$
\int_{\tilde{B}}|\nabla u|^{2}+H^{2}(P \cap \tilde{B}) \leq \int_{\tilde{B}}|\nabla \tilde{v}|^{2}+H^{2}(\tilde{\Gamma} \cap \tilde{B}) .
$$

In the other hand $u$ is equal to $\tilde{v}$ in $\tilde{B} \backslash \mathcal{C}$ and it is the same for $\Gamma$ and $\tilde{\Gamma}$. We deduce

$$
\int_{\mathcal{C}}|\nabla u|^{2} d x d y d z+H^{2}(P \cap \mathcal{C}) \leq \int_{\mathcal{C}}|\nabla \tilde{v}|^{2} d x d y d z+H^{2}(\tilde{\Gamma} \cap \mathcal{C}) .
$$

Now, since $u$ and $\tilde{v}$ are vertically constant, $\nabla_{z} u=\nabla_{z} \tilde{v}=0$, and $\nabla_{x} u, \nabla_{y} u$ are also constant with respect to the variable $z$ (as for $\tilde{v}$ ). Thus

$2 R \int_{B}|\nabla u(x, y, 0)|^{2} d x d y+H^{2}(P \cap \mathcal{C}) \leq 2 R \int_{B}|\nabla v(x, y)|^{2} d x d y+H^{2}(\tilde{\Gamma} \cap \mathcal{C})$.

To conclude we will use the following lemma.

LEMma 25. If $\Gamma$ is rectifiable and contained in a plane $Q$ then

$$
H^{2}(\Gamma \times[-R, R])=2 R H^{1}(\Gamma) .
$$

Proof. We will use the coarea formula (see Theorem 2.93 of [3]). We take $f: \mathbb{R}^{3} \rightarrow \mathbb{R}$ the orthogonal projection on the coordinate orthogonal to $Q$. By this way, if $E:=\Gamma \times[-R, R]$, we have $E \cap f^{-1}(t)=\Gamma$ for all $t \in[-R, R] . E$ is rectifiable (because $\Gamma$ is by hypothesis). So we can apply the coarea formula. To do this we have to calculate the jacobian $c_{k} d^{E} f_{x}$. By construction, the approximate tangente plane in each point of $E$ is orthogonal to $Q$. We deduce that if $T_{x}$ is a tangent plane, then there is a basis of $T_{x}$ $\left(\overrightarrow{b_{1}}, \overrightarrow{b_{2}}\right)$ such that $\overrightarrow{b_{1}}$ is orthogonal to $Q$. Since the function $f$ is the projection on $\overrightarrow{b_{1}}$, and its derivative as well (because $f$ is linear ) we obtain that the matrix of $d^{E} f_{x}: T_{x} \rightarrow \mathbb{R}$ in the basis $\left(\overrightarrow{b_{1}}, \overrightarrow{b_{2}}\right)$ is

$$
d^{E} f_{x}=(1,0)
$$

thus

$$
c_{k} d^{E} f_{x}=\sqrt{\operatorname{det}\left[(1,0){ }^{t}(1,0)\right]}=1 .
$$

Therefore

$$
H^{2}(E)=\int_{-R}^{R} H^{1}(\Gamma)=2 R H^{1}(\Gamma)
$$


Here we can suppose that $\Gamma$ is rectifiable. Indeed, the definition of Mumford-Shah minimizers is equivalent if we only allow rectifiables competitors. This is because the jump set of a $S B V$ function is rectifiable and in [11] it is proved that the relaxed functional on the $S B V$ space has same minimizers.

So we have

$$
\begin{aligned}
& 2 R \int_{B}|\nabla u(x, y, 0)|^{2} d x d y+2 R H^{1}(P \cap B) \\
& \leq 2 R \int_{B}|\nabla v(x, y)|^{2} d x d y+2 R H^{1}(\Gamma \cap B)+H^{2}(B \times\{ \pm R\}) .
\end{aligned}
$$

Then, dividing by $2 R$,

$$
\int_{B}|\nabla u(x, y, 0)|^{2} d x d y+H^{1}(P \cap B) \leq \int_{B}|\nabla v(x, y)|^{2} d x d y+H^{1}(\Gamma \cap B)+\pi \frac{\rho^{2}}{R}
$$

thus, letting $R$ go to infinity,

$$
\int_{B}|\nabla u(x, y, 0)|^{2} d x d y+H^{1}(P \cap B) \leq \int_{B}|\nabla v(x, y)|^{2} d x d y+H^{1}(\Gamma \cap B) .
$$

This last inequality proves that $\left(u(x, y, 0), P_{0}\right)$ is a global minimizer in $\mathbb{R}^{2}$, and since $P_{0}$ is a half-line, $u$ is a cracktip.

REMARK 26. Using a similar argument as the preceding proof, we can show that the first eigenvalue for $-\Delta$ in $S^{2} \backslash P$ with Neumann boundary conditions (where $P$ is still a half-plane), is equal to $\frac{3}{4}$. Moreover we can prove that the eigenspace is of dimension 1 , generated by a function of type cracktip $\times \mathbb{R}$, thus we have a new proof of Proposition 22. The argument is to take an eigenfunction $f$ in $S^{2} \backslash P$, then to consider $u(x):=\|x\|^{\alpha} f\left(\frac{x}{\|x\|}\right)$ with a good coefficient $\left.\alpha \in] ; 0, \frac{1}{2}\right]$ that makes $u$ harmonic. Finally we use the same sort of estimates on the harmonic measure to prove that $u$ is vertically constant. Thus we have reduced the problem in dimension 2 and we conclude using that we know the eigenfunctions on the circle. A detailed proof is done in [15]. 


\section{Open questions.}

As it is said in the introduction, this paper is a very short step in the discovering of all the global minimizers in $\mathbb{R}^{N}$. This final goal seems rather far but nevertheless some open questions might be accessible in a more reasonable time. All the following questions were pointed out by Guy David in [8], and unfortunately they are still open after this paper.

- Suppose that $(u, K)$ is a global minimizer in $\mathbb{R}^{N}$. Is it true that $K$ is conical?

- Suppose that $(u, K)$ is a global minimizer in $\mathbb{R}^{N}$, and $K$ is a cone. Is it true that $\frac{3-2 N}{4}$ is the smallest eigenvalue of the Laplacian on $S^{N-1} \backslash K$ ?

- Suppose that $(u, K)$ is a global minimizer in $\mathbb{R}^{3}$, and suppose that $K$ is contained in a plan (and not empty). Is it true that $K$ is a plane or a halfplane?

- Could one found an extra global minimizer in $\mathbb{R}^{3}$ by blowing up the minimizer described in section 76.c. of [8] (see also [17])?

One can find other open questions on global minimizers in the last page of [8].

Acknowledgments. The author wishes to thank Guy David for having introduced him to the Mumford-Shah Functional, and for many helpful and interesting discussions on this subject.

\section{REFERENCES}

[1] R. A. Adams, Sobolev spaces. Academic Press [A subsidiary of Harcourt Brace Jovanovich, Publishers], Pure and Applied Mathematics, Vol. 65 (New YorkLondon, 1975).

[2] L. Ambrosio - N. Fusco - D. Pallara, Partial regularity of free discontinuity sets. II. Ann. Scuola Norm. Sup. Pisa Cl. Sci. (4), 24 (1) ( 1997), pp. 39-62.

[3] L. Ambrosio - N. Fusco - D. PALlara, Functions of bounded variation and free discontinuity problems. Oxford Mathematical Monographs. The Clarendon Press Oxford University Press (New York, 2000).

[4] A. Bonnet, On the regularity of edges in image segmentation. Ann. Inst. H. Poincaré Anal. Non Linéaire, 13 (4) (1996), pp. 485-528.

[5] M. DAuge, Elliptic boundary value problems on corner domains, volume 1341 of Lecture Notes in Mathematics. Smoothness and asymptotics of solutions (Springer-Verlag, Berlin, 1988).

[6] M. DAuge, Neumann and mixed problems on curvilinear polyhedra. Integral Equations Operator Theory, 15 (2) (1992), pp. 227-261. 
[7] G. DAVID, $C^{1}$-arcs for minimizers of the Mumford-Shah functional. SIAM J. Appl. Math., 56 (3) (1996), pp. 783-888.

[8] G. DAvID, Singular sets of minimizers for the Mumford-Shah functional, volume 233 of Progress in Mathematics (Birkhäuser Verlag, Basel, 2005).

[9] G. DAVID, Hölder regularity of two dimensional almost-minimal sets in $\mathbb{R}^{n}$. Geom. Funct. Anal., 18 (4) (2008), pp. 1168-1235.

[10] G. David - D. P. Thierry - T. Toro, A generalisation of Reifenberg's theorem in $\mathbb{R}^{3}$. Ann. Fac. Sc. Toul. (6), 18 (1) (2009), pp. 65-246.

[11] E. De Giorgi - M. CARriero - A. LeACI, Existence theorem for a minimum problem with free discontinuity set. Arch. Rational Mech. Anal., 108 (3) (1989), pp. 195-218.

[12] D. Gilbarg - N. S. Trudinger, Elliptic partial differential equations of second order, volume 224 of Grundlehren der Mathematischen Wissenschaften [Fundamental Principles of Mathematical Sciences]. (Springer-Verlag, Berlin, second edition, 1983).

[13] J. B. Keller, Singularities at the tip of a plane angular sector. J. Math. Phys., 40 (2) (1999), pp. 1087-1092.

[14] V. A. KozLOV - V. G. MaZ'YA - J. Rossmann, Spectral problems associated with corner singularities of solutions to elliptic equations, volume 85 of Mathematical Surveys and Monographs. American Mathematical Society, Providence, RI, 2001.

[15] A. Lemenant, Sur la régularité des minimiseurs de Mumford-Shah en dimension 3 et supérieure. Thesis Université Paris Sud XI (Orsay, 2008).

[16] P. LÉVY-BruHL, Introduction à la théorie spectrale. Dunod, 2003.

[17] Benoît Merlet, Numerical study of a new global minimizer for the Mumford-Shah functional in $\boldsymbol{R}^{3}$. ESAIM Control Optim. Calc. Var., 13 (3) (2007), pp. 553-569.

[18] D. Mumford - J. SHAH, Optimal approximations by piecewise smooth functions and associated variational problems. Comm. Pure Appl. Math., 42, (5) (1989), pp. 577-685.

[19] M. REeD - B. Simon, Methods of modern mathematical physics. IV. Analysis of operators. Academic Press [Harcourt Brace Jovanovich Publishers], (New York, 1978).

[20] J. E. TAYLOR, The structure of singularities in soap-bubble-like and soapfilm-like minimal surfaces. Ann. of Math. (2), 103 (3) (1976), pp. 489-539.

[21] M. E. TaYlor, Partial Differential Equations Basic Theory, Vol. 23 of Texts in Applied Mathematics. (Springer, 1st ed. 1996). (Corr. 2nd printing, 1999).

Manoscritto pervenuto in redazione il 24 settembre 2008. 
